\title{
BMJ Global Health Are COVID-19 mitigation measures reducing preterm birth rate in China?
}

\author{
Zheng Bian, Xiaoxian Qu, Hao Ying, Xiaohua Liu
}

\begin{abstract}
To cite: Bian Z, Qu X, Ying $\mathrm{H}$, et al. Are COVID-19 mitigation measures reducing preterm birth rate in China?BMJ Global Health 2021;6:e006359. doi:10.1136/ bmjgh-2021-006359
\end{abstract}

Handling editor Seye Abimbola

- Additional supplemental material is published online only. To view, please visit the journal online (http://dx.doi.org/10. 1136/bmjgh-2021-006359).

$\mathrm{HY}$ and XL contributed equally.

$Z B$ and $X Q$ are joint first authors

Received 22 May 2021

Accepted 29 June 2021

\section{Check for updates}

\section{(c) Author(s) (or their}

employer(s)) 2021. Re-use permitted under CC BY-NC. No commercial re-use. See rights and permissions. Published by BMJ

Department of Obstetrics, Shanghai First Maternity and Infant Hospital, Tongji University School of Medicine, Tongji University, Shanghai, China

Correspondence to

Dr Xiaohua Liu;

annaabcd114@hotmail.com

\section{ABSTRACT}

Objective Preterm birth is the leading cause of child morbidity and mortality globally. We aimed to determine the impact of the COVID-19 mitigation measures implemented in China on 23 January 2020 on the incidence of preterm birth in our institution.

Design Logistic regression analysis was used to investigate the association between the national COVID-19 mitigation measures implemented in China and the incidence of preterm birth.

Setting Shanghai First Maternity and Infant Hospital, Shanghai China.

Participants All singleton deliveries abstracted from electronic medical record between 1 January 2014 to 31 December 2020.

Main outcome measures Preterm birth rate.

Results Data on 164107 singleton deliveries were available. COVID-19 mitigation measures were consistently associated with significant reductions in preterm birth in the 2-month, 3-month, 4-month, 5-month time windows after implementation (+2 months, 0R $0.80,95 \% \mathrm{Cl} 0.69$ to $0.94 ;+3$ months, OR $0.83,95 \% \mathrm{Cl} 0.73$ to $0.94 ;+4$ months, OR $0.82,95 \% \mathrm{Cl} 0.73$ to $0.92 ;+5$ months, OR $0.84,95 \% \mathrm{Cl} 0.76$ to 0.93$)$. These reductions in preterm birth were obvious across various degrees of prematurity, but were statistically significant only in moderate-to-late preterm birth (32 complete weeks to 36 weeks and 6 days) subgroup. The preterm birth difference disappeared gradually after various restrictions were removed (7th12th month of $2020,0 \mathrm{R} 1.02,95 \% \mathrm{Cl} 0.94$ to 1.11). There was no difference in stillbirth rate across the study time window.

Conclusion Substantial decreases in preterm birth rates were observed following implementation of the national COVID-19 mitigation measures in China. Further study is warranted to explore the underlying mechanisms associated with this observation.

\section{INTRODUCTION}

Wuhan, China, the first place to report COVID-19 contagious disease, was placed in lockdown status on 23 January 2020 by the Chinese government. The whole country then entered level 1 emergency. Measures were taken to prevent the spread of infection and mitigate its population flow and contact. The COVID-19 pandemic, the intensity and immediacy of the policy responses were

\section{WHAT IS ALREADY KNOWN? \\ $\Rightarrow$ We are aware of several studies, mostly originating from high-income countries, that explore the associ- ation of lockdowns implemented in response to the COVID-19 pandemic and rates of preterm birth or low birth weight. \\ $\Rightarrow$ The conclusions from these studies were conflict- ing, those suggesting that lockdown was associated with decreased rates of preterm birth or low birth weight and those that conclude it was not. \\ $\Rightarrow$ COVID-19 mitigation measures have been im- plemented by different countries with substan- tial variation in timing, content, strictness and comprehensiveness.}

\section{WHAT ARE THE NEW FINDINGS?}

$\Rightarrow$ China was the first country to experience the pandemic and take the strict comprehensive mitigation measures, however, daily life in Shanghai, China returned to normal by 18 May 2020 .

$\Rightarrow$ The mitigation time window provides a unique opportunity to evaluate their effects on preterm.

$\Rightarrow$ In this large study with 164107 singleton births, substantial reductions in preterm births were observed following implementation of the national COVID-19 mitigation measures in China.

$\Rightarrow$ The reductions in preterm birth disappeared after daily life and work returned back to normal.

\section{WHAT DO THE NEW FINDINGS IMPLY?}

$\Rightarrow$ Our study progressed substantially from earlier studies that we also had the preterm information after the mitigation measures when life and work was back to normal in Chinese population, thus strengthening causal interpretation of the observed association between the COVID-19 mitigation measures and decreased preterm birth rates, which may in turn provide an opportunity to identify novel preventive strategies for preterm birth.

$\Rightarrow$ Such efforts could provide new opportunities for preterm birth prevention with substantial implications on global perinatal and public health.

having an unprecedented impact on medical services and public society. China was the first country to experience the pandemic and take the strict comprehensive mitigation measures, however, daily life in China returned to normal by 18 May 2020 when schools and 
childcare facilities, restaurant and entertainment places were reopened. ${ }^{1}$ The mitigation time window provides a unique opportunity to evaluate their effects on some obstetric issues such as preterm birth as a natural experiment.

WHO has estimated $>1$ in 10 births is preterm. While Asian individuals have a relatively low incidence of preterm birth, China still has the second largest absolute number of preterm births in the world. ${ }^{2}$ Preterm birth is the primary contributor to perinatal morbidity and mortality. Additionally, preterm birth survivors and their families frequently withstand long-term adverse consequences such as cerebral palsy. ${ }^{34}$ Relatively few cases of preterm birth can be prevented using currently available strategies because the aetiology of spontaneous preterm birth is multifactorial. ${ }^{5}$

Both Denmark and Ireland studies have provided evidence indicating significant reductions in the number of extremely preterm and very low birthweight births following implementation of national COVID-19 mitigation measures. ${ }^{67}$ Also, the Netherlands study reported the decrease of moderate-to-late preterm incidence. ${ }^{8}$ However, the study from Philadelphia, Pennsylvania, found no change in preterm birth or stillbirths associated with the lockdown. ${ }^{9}$ COVID-19 mitigation measures have been implemented by different countries with substantial variation in timing, content, intensity and comprehensiveness. Furthermore, various risk factors for preterm birth that might be responsive to lockdown measures also vary across populations. As such, international data from various population are important to further study the association between COVID-19 mitigation measures and preterm births, to explore differential impact between spontaneous and induced preterm deliveries and to identify the potential underlying mechanisms.

\section{METHODS}

\section{Study design and population}

We performed logistic regression analysis to investigate the association between the national implementation of COVID-19 mitigation measures and the incidence of preterm birth in Shanghai First Maternity and Infant Hospital (SFMIH), Tongji University School of Medicine. We examined data from all pregnant women who received care at SFMIH from 1 January 2014 to 31 December 2020. SFMIH is now the largest obstetric care centre in Shanghai, as well as in China. According to Shanghai guidelines, women with uncomplicated pregnancies are offered at least 9-10 antenatal visits, the first one occurring between 8 and 9 weeks of gestation when gestational age is determined by ultrasound examination. ${ }^{10}$

Inclusion criteria for the present study included all singleton pregnancy. Multiple births were excluded from the analysis due to the more complicated aetiologies and increased risk of preterm birth. We also excluded infants delivered prior to 24 complete weeks because China national perinatal guidelines advise no active management for infants of $<24$ complete weeks. Thus, the preterm is defined as birth that occurs after 24 weeks and before the completion of 37 gestational weeks. We further divided preterm birth into spontaneous preterm due to premature parturitional process, and iatrogenic preterm because the health of the mother or fetus was at risk (eg, with major haemorrhage, hypertension or poor fetal growth). Small for gestational age (SGA) and large for gestational age (LGA) were defined based on Asian sex-specific and gestational age-specific birth weight reference curves. ${ }^{11}$

\section{Data collection and covariates}

The information engineer and study group abstracted and extracted data from the hospital electronic medical records based on criteria set forth on the standardised form for data collection. The abstracted information from medical records included maternal demographic characteristics, medical and surgical history, obstetric complications, type of conception (natural or in vitro fertilization and embryo transfer assisted), labour and delivery summaries, postpartum maternal and neonatal information. Based on review of the literature on preterm, the following covariates were considered as potential confounders: prepregnant body mass index (PPBMI), maternal age, education level, insurance status, marriage status, conception type, gender of fetus, maternal chronic medical conditions and obstetric complications including hypertensive disorders, pregestational diabetes and gestational diabetes (DM and GDM) and intrahepatic cholestasis of pregnancy (ICP).

\section{Statistical analysis}

A single large dataset was created with deidentified annual data collected from medical records. The research group examined data for missing and extreme values and logic check. Descriptive statistics included numbers and percentages for categoric variables, means and $\mathrm{SD}$ for continuous variables. We assessed the differences between two groups using $\chi^{2}$ test for dichotomous variables and Student's t-test for continuous outcomes. The association between the national COVID-19 mitigation measures and the incidence of preterm births was studied using logistic regression analysis. In the primary analyses, we studied the overall incidence of preterm birth (ie, number of neonates born at a gestational age of $<37$ complete weeks). In additional stratified analyses, we assessed whether there were differential impact of mitigation measures on preterm birth rates according to the degree of prematurity: 24 complete weeks to 31 weeks and 6 days, and 32 complete weeks to 36 weeks and 6 days. To explore the regression continuity in preterm birth incidence, we analysed spontaneous or iatrogenic preterm birth separately surrounding the implementation of mitigation measures.

We assessed six time windows in separate analyses: 1 month, 2 months, 3 months, 4 months and 5 months after dates of implementation (23 January 2020) and the last 
half year of 2020 when mitigation measures were relaxed (from July 2020 to December 2020). We compared the period after implementation of the measures in 2020 to the exact same time periods in each year preceding the COVID-19 pandemic (2014-2019). Logistic regression was used to determine the ORs and $95 \%$ CIs of preterm while adjusting for confounders. Based on review of the literature, the following covariates were considered as potential confounders or effect modifiers: maternal age, PPBMI, education, insurance status, type of conception, parity, maternal chronic medical conditions, pregnancy complications, gender of fetus. All data were analysed with SPSS software (V.22; IBM, Chicago, Illinois, USA). A probability value of $<0.05$ was considered to be statistically significant.

We used these relatively short time windows to exclude other interventions or major impacts and assumed that any change observed in preterm birth was due to the COVID-19 mitigation measures. Furthermore, we studied the preterm incidence when mitigation measures were relaxed (from July 2020 to December 2020) to explore whether the preterm rates would return to baseline level without the influence of the measures.

\section{RESULTS}

We abstracted data on 169073 deliveries. None of these women was infected with COVID-19. After excluding 4937 multiple pregnancies and 29 deliveries prior to 24 gestational weeks, 164107 singleton deliveries were included in our analysis. More than $99 \%$ of the women in our research population were of Han ethnicity. Smoking or alcohol use was very unusual in this population (data not shown). Other demographic baseline and obstetric characteristics of the study subjects are shown in table 1 . The women delivered in 2020 were complicated with higher incidences of hypertensive disorders and ICP, but lower incidences of diabetes. The early preterm rate $(<32$ weeks) was lower in 2020, and more LGA and less SGA babies were delivered in this year. Women were more likely to undergo caesarean delivery in 2020. While there were statistically significant differences between groups with regard to BMI, insurance, parity and education, the differences were not clinically meaningful because of the large sample size.

Online supplemental figure 1 depicts the annual trends in preterm birth rates over time (2014-2020). The preterm birth rates were generally stable during the study period with rates of $5.2 \%-5.6 \%$. A clear discontinuity of preterm rate in the regression lines was observed for the COVID-19 mitigation measures introduced on 23 January 2020 (figure 1). In additional stratified analyses, we could determine the discontinuity mainly stemmed from spontaneous preterm (figure 1) and moderate-to-late preterm birth (32 complete weeks to 36 weeks and 6 days, figure 2). The rate of iatrogenic preterm was relatively constant surrounding implementation of measures to reduce the spread of the virus. Accordingly, the lockdown
Table 1 Baseline and obstetric characteristics of the study subjects $(n=164107)$

\begin{tabular}{|c|c|c|}
\hline Characteristic & $\begin{array}{l}2020(n=24 \\
312)\end{array}$ & $\begin{array}{l}2014-2019 \\
(n=139795)\end{array}$ \\
\hline \multicolumn{3}{|l|}{ Maternal age, years-\% } \\
\hline$\geq 35.0$ & 18.8 & 16.1 \\
\hline $25.0 \sim 34.9$ & 78.6 & 80.0 \\
\hline$<25.0$ & 2.6 & 3.9 \\
\hline Insurance-\% & 73.4 & 71.3 \\
\hline Married-\% & 99.2 & 99.2 \\
\hline Nulliparity-\% & 73.9 & 74.8 \\
\hline \multicolumn{3}{|l|}{ BMI at first prenatal visit- \% } \\
\hline$\geq 30.0$ & 1.8 & 1.5 \\
\hline $25.0-29.9$ & 12.5 & 11.9 \\
\hline 20.0-24.9 & 57.3 & 57.8 \\
\hline$<20.0$ & 28.4 & 28.8 \\
\hline Education-years $($ mean $\pm S D)$ & $15.3 \pm 2.8$ & $14.9 \pm 2.6$ \\
\hline Assisted conception-\% & 2.9 & 3.2 \\
\hline $\mathrm{DM}$ and $\mathrm{GDM}-\%$ & 11.3 & 12.3 \\
\hline Hypertensive disorders - \% & 5.8 & 5.4 \\
\hline $\mathrm{ICP}-\%$ & 1.2 & 1.0 \\
\hline \multicolumn{3}{|l|}{ Gestational age at delivery-\% } \\
\hline$<32$ GW & 0.5 & 0.7 \\
\hline $32-36 \mathrm{GW}$ & 4.7 & 4.7 \\
\hline$\geq 37 \mathrm{GW}$ & 94.8 & 94.6 \\
\hline Stillbirth-\% & 0.2 & 0.2 \\
\hline \multicolumn{3}{|l|}{ Birth weight-\% } \\
\hline Large for gestational age & 4.7 & 4.5 \\
\hline Average for gestational age & 92.4 & 92.3 \\
\hline Small for gestational age & 2.9 & 3.2 \\
\hline \multicolumn{3}{|l|}{ Delivery mode-\% } \\
\hline Spontaneous delivery & 54.5 & 60.6 \\
\hline Assisted vaginal delivery & 1.5 & 1.3 \\
\hline Caesarean delivery & 44.0 & 38.1 \\
\hline
\end{tabular}

BMI, body mass index; DM, pregestational diabetes; GDM, gestational diabetes; GW, gestational week; ICP, intrahepatic cholestasis of pregnancy.

measures were consistently associated with significant reductions in preterm birth in the 2-month, 3-month, 4-month, 5-month time windows after implementation (+2 months, OR $0.80,95 \%$ CI 0.69 to $0.94 ;+3$ months, OR $0.83,95 \%$ CI 0.73 to $0.94 ;+4$ months, OR $0.82,95 \%$ CI 0.73 to $0.92 ;+5$ months, OR $0.84,95 \%$ CI 0.76 to 0.93 , table 2). These reductions in preterm births were obvious across various degrees of prematurity, but were statistically significant only in moderate-to-late preterm birth (32 complete weeks to 36 weeks and 6 days) subgroup (table 2). The preterm difference disappeared when mitigation measures were relaxed (7th-12th month of 


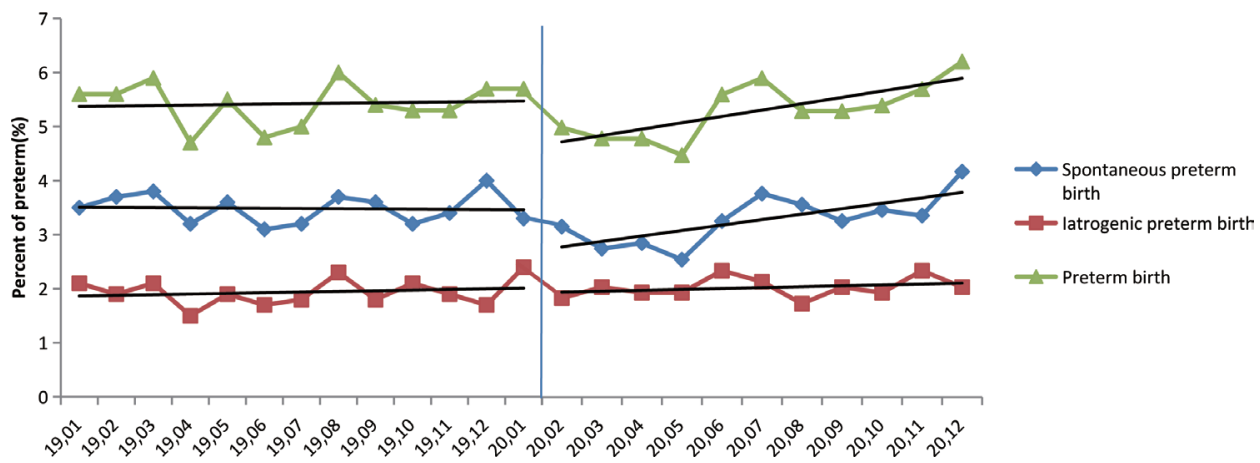

Figure 1 Regression discontinuity in monthly preterm birth incidence surrounding implementation of COVID-19 mitigation measures.

2020, OR 1.02, 95\% CI 0.94 to 1.11). There was no difference in stillbirth rate across the study time window.

\section{DISCUSSION}

In this large study with 164107 singleton births, we observed substantial decreases in preterm birth rates following implementation of the national COVID-19 lockdown measures in China. These decreases were consistent across early to late preterm birth. The reductions in preterm birth disappeared after daily life and work returned back to normal. Our study adds important evidence to the existing data on this subject and confirms the observation from some other studies that mitigation measures are associated with preterm birth reduction, which may provide an opportunity to identify novel preventive strategies for preterm birth.

Our large study assessed the impact of COVID-19 mitigation measures implemented in China on the incidence of preterm birth with data abstracted directly from electronic medical records, which was different from the Netherlands and Danish studies using blood spot screening programme. ${ }^{6}$ Our dataset had individual-level information on relevant covariates, including demographic information, parity, medical and obstetric complications, induction and delivery information, therefore, we could minimise the influence of changes in demographic composition of the population following mitigation measures (eg, through the mitigation of population flow), which might have contributed to the findings. Also, our analysis was able to explore differential impact between spontaneous and iatrogenic preterm deliveries. By using the same quasi-experimental approach as the Netherlands study did, our study progressed substantially from earlier studies that we also had the preterm information after the mitigation measures when life and work was back to normal in Chinese population, thus strengthening causal interpretation of the observed association between the COVID-19 mitigation measures and reduced preterm birth incidences.

Our study also has some limitations. The unanticipated nature of the COVID-19 pandemic and associated mitigation measures allows for a retrospective analysis and makes randomised assignment or a prospective cohort approach impossible. The rate of preterm birth increased in June right after the COVID-19 restrictions being eased (18 May). We noticed the increase in June was masked in the logistic regression analysis by overlapping windows. This increases might be explained by seasonal influence as the preterm rate also increased in summer in previous year as shown in figure 1 . We used these relatively short time windows to exclude major influences as season. The $1 / 6 \sim 1 / 5$ of babies in Shanghai have been delivered in SFMIH in past 8 years, thus, the present study population is a good representative sample of Shanghai and developed areas in China, but our results may not be generalisable to undeveloped areas in China.

Consistent with the Netherlands study, we observed a significant reduction of moderate-to-late preterm (ie, 32 complete weeks to 36 weeks and 6 days), whereas the decrease was fairly constant in early preterm even though

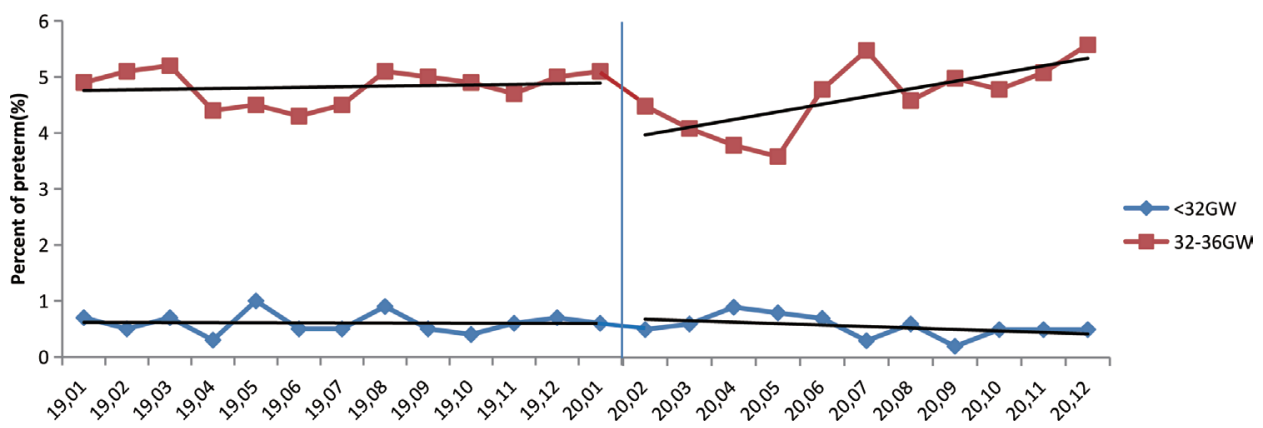

Figure 2 Regression discontinuity in monthly different prematurity incidence surrounding implementation of COVID-19 mitigation measures. GW, gestational week. 


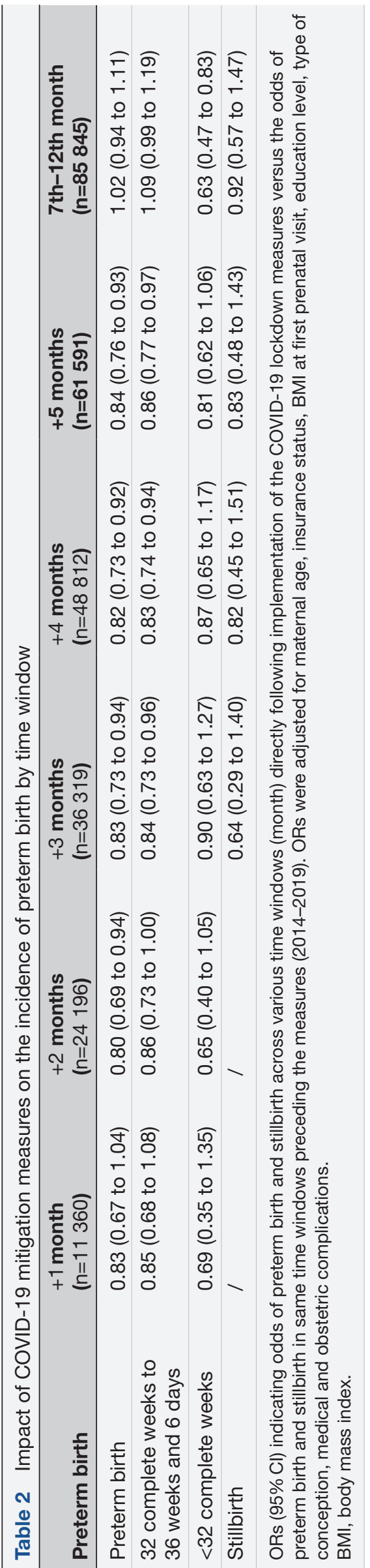

the result was not statistically significant due to the insufficient cases in this subgroup. In the Irish study, rates of extremely low birthweight and very low birthweight births were lower between 1 January 2020 and 30 April 2020, than in the same period in the preceding 19 years. ${ }^{7}$ Only one extremely preterm birth had been observed in the Danish study using national blood spot screening data in the first month following COVID-19 lockdown, whereas five to six were calculated. The observed reduction in preterm births in Denmark and Ireland predominantly affected the smallest babies, and the numbers were small. ${ }^{67}$ The information on stillbirths was unavailable in these two studies, thus one cannot discern whether a part of the observed reduction in very early preterm births occurred at the expense of an increase in stillbirths. A study in a London (UK) hospital compared birth outcomes before and after the outbreak of COVID-19 pandemic revealed no changes in the incidence of births prior to 34 or 37 gestational weeks. ${ }^{12}$ The authors observed an increase in stillbirths of six per 1000 births following the pandemic. However, this study had a small sample size and it did not specifically investigate the effect of the mitigation measures. Importantly, we did not observe an increase in the stillbirth rate during the COVID-19 pandemic. The study from Philadelphia, Pennsylvania, found no change in preterm birth or stillbirths associated with the lockdown. ${ }^{9}$ The differences between studies may be due to varieties in the strictness and comprehensiveness of lockdown measures, enforcement of lockdown orders, access to healthcare or financial and social stressors.

Countless studies have been carried to clarify the aetiology of spontaneous preterm and to explore effective preventive strategies. Efforts have been largely disappointing because the aetiology of prematurity is complex. ${ }^{13}$ The COVID-19 mitigation measures might affect some known risk factors for preterm birth. These risk factors include asymptomatic infection and inflammation which can cause intrauterine infection and inflammation through vertical transmission, initiating a succession of mechanism resulting in preterm birth. Wearing masks, keeping physical distance and staying at home, closing of schools and childcare facilities, lack of population flow and increased awareness of hygiene (eg, hand washing and window opening) all reduce contact with pathogens and minimise the risk of some infections. It had been reported that common infectious disease morbidity decreased during the COVID-19 emergency response, thus reducing overall inflammatory state of pregnant women. ${ }^{14}$ The literature on a potential association between work and premature birth however is contradictory. ${ }^{15}$ The potential reduced physical demands associated with less work, less travel and even reductions in minor transportation accidents could all be possible contributors. Women were more likely to prepare food at home as opposed to eating in a restaurant which may have improved nutrition and working from home may have also optimised sleep duration, which could all have a positive influence on prematurity rates. ${ }^{16}$ Substantial 
reductions in air pollution have also been reported following COVID-19 mitigation measures, including in China. ${ }^{17}$ Given exposure to air pollution is the recognised increased risk of delivering preterm, this finding could also explain the observed reductions. ${ }^{18}$ The short timing window of the observed preterm birth reductions in our study suggests that hygiene measures and behavioural changes related to mitigation measures might have been instrumental.

Approximately one-third preterm births are iatrogenic, usually for maternal or fetal health concerns. ${ }^{19}$ Changes in obstetric practice or care-seeking behaviour of pregnant women might also have contributed induced preterm delivery rates. In our dataset, we did observed a slightly higher incidences of hypertensive disorders and ICP in 2020 which might increase preterm deliveries, however the iatrogenic preterm rate was generally constant over the study period, and the preterm reduction associated with mitigation measures we observed was primarily among spontaneous preterm births.

\section{CONCLUSION}

In summary, we have reported that national implementation of COVID-19 mitigation measures in China was associated with a substantial reduction in preterm births, adding new evidence to preliminary findings from other countries and generating the hypothesis that the COVID-19 mitigation measures could reduce preterm births. Identification of the underlying mechanisms is an essential next step, which could be vital to developing novel preventive strategies for preterm birth.

Contributors $\mathrm{ZB}$ and $\mathrm{XQ}$ mainly contributed to design and critical writing. $\mathrm{HY}$ and $X L$ mainly contributed to design, the critical revision, interpretation of the data and the final approval of the version to be published.

Funding This study was funded by the National Natural Science Foundation of China (No. 82071678), the Key Research Project of Pudong New Area Population and Family Planning Commission (No. PW2020E-1), Clinical Research Plan of SHDC (No. SHDC2020CR2059B). There are no competing interest.

Competing interests None declared.

Patient consent for publication Not required.

Ethics approval This research proposal was approved by the ethics review board at Shanghai First Maternity and Infant Hospital, Shanghai China (Date: 12 February 2021, reference number: KS 21409).

Provenance and peer review Not commissioned; externally peer reviewed.

Data availability statement Data are available on reasonable request. Deidentified participant dataData are available upon request from Dr Liu XiaohuaEmail: annaabcd114@hotmail.com.

Supplemental material This content has been supplied by the author(s). It has not been vetted by BMJ Publishing Group Limited (BMJ) and may not have been peer-reviewed. Any opinions or recommendations discussed are solely those of the author(s) and are not endorsed by BMJ. BMJ disclaims all liability and responsibility arising from any reliance placed on the content. Where the content includes any translated material, BMJ does not warrant the accuracy and reliability of the translations (including but not limited to local regulations, clinical guidelines, terminology, drug names and drug dosages), and is not responsible for any error and/or omissions arising from translation and adaptation or otherwise.

Open access This is an open access article distributed in accordance with the Creative Commons Attribution Non Commercial (CC BY-NC 4.0) license, which permits others to distribute, remix, adapt, build upon this work non-commercially, and license their derivative works on different terms, provided the original work is properly cited, appropriate credit is given, any changes made indicated, and the use is non-commercial. See: http://creativecommons.org/licenses/by-nc/4.0/.

\section{REFERENCES}

1 Zhao K, Long C, Wang Y, et al. Negligible risk of the COVID-19 resurgence caused by work resuming in China (outside Hubei): a statistical probability study. J Public Health 2020;42:651-2.

2 Chawanpaiboon S, Vogel JP, Moller A-B, et al. Global, regional, and national estimates of levels of preterm birth in 2014: a systematic review and modelling analysis. Lancet Glob Health 2019;7:e37-46.

3 Twilhaar ES, Wade RM, de Kieviet JF, et al. Cognitive outcomes of children born extremely or very preterm since the 1990s and associated risk factors: a meta-analysis and meta-regression. JAMA Pediatr 2018;172:361-7.

4 Vollmer B, Stålnacke J. Young adult motor, sensory, and cognitive outcomes and longitudinal development after very and extremely preterm birth. Neuropediatrics 2019;50:219-27.

5 Matei A, Saccone G, Vogel JP, et al. Primary and secondary prevention of preterm birth: a review of systematic reviews and ongoing randomized controlled trials. Eur J Obstet Gynecol Reprod Biol 2019;236:224-39.

6 Hedermann G, Hedley PL, Bækvad-Hansen M, et al. Danish premature birth rates during the COVID-19 lockdown. Arch Dis Child Fetal Neonatal Ed 2021;106:93-95.

7 Philip RK, Purtill H, Reidy E. Reduction in preterm births during the COVID-19 lockdown in Ireland: a natural experiment allowing analysis of data from the prior two decades. medRxiv 2020.

8 Been JV, Burgos Ochoa L, Bertens LCM, et al. Impact of COVID-19 mitigation measures on the incidence of preterm birth: a national quasi-experimental study. Lancet Public Health 2020;5:e604-11.

9 Handley SC, Mullin AM, Elovitz MA, et al. Changes in preterm birth phenotypes and stillbirth at 2 Philadelphia hospitals during the SARS-CoV-2 pandemic, March-June 2020. JAMA 2021;325:87-89.

10 Zhao Q, Huang ZJ, Yang S, et al. The utilization of antenatal care among rural-to-urban migrant women in Shanghai: a hospital-based cross-sectional study. BMC Public Health 2012;12:1012.

11 Buck Louis GM, Grewal J, Albert PS, et al. Racial/Ethnic standards for fetal growth: the NICHD fetal growth studies. Am J Obstet Gynecol 2015;213:449.e1-449.e41.

12 Khalil A, von Dadelszen P, Draycott T, et al. Change in the incidence of stillbirth and preterm delivery during the COVID-19 pandemic. JAMA 2020;324:705-6.

13 Goldenberg RL, Culhane JF, lams JD, et al. Epidemiology and causes of preterm birth. Lancet 2008;371:75-84.

14 Lyu HX, Cheng HB, Huang XY. Analysis of changes of common infectious diseases morbidity during COVID-19 emergency response in Longhua district from Shenzhen. Journal of Preventive Medicine Information 2020;36:1404-9.

15 Snijder CA, Brand T, Jaddoe V, et al. Physically demanding work, fetal growth and the risk of adverse birth outcomes. the generation $\mathrm{R}$ study. Occup Environ Med 2012;69:543-50.

16 Yang G-Y, Lin X-L, Fang A-P, et al. Eating habits and lifestyles during the initial stage of the COVID-19 Lockdown in China: a crosssectional study. Nutrients 2021;13:970.

17 Mühlberg B. Coronavirus response leads to big drop in air pollution. NL times, 2020. Available: https://nltimes.nl/2020/03/27/coronavirusresponse leads-big-drop-air-pollution

18 Bekkar B, Pacheco S, Basu R, et al. Association of air pollution and heat exposure with preterm birth, low birth weight, and stillbirth in the US: a systematic review. JAMA Netw Open 2020;3:e208243.

19 Chen C, Zhang JW, Xia HW, et al. Preterm birth in China between 2015 and 2016. Am J Public Health 2019;109:1597-604. 